\title{
Influencia de la personalidad en el dolor cervical postraumático
}

\section{Influence of the personality in the cervical posttraumatic pain}

\begin{abstract}
Resumen
El dolor cervical postraumático es una de las afecciones más comunes en la consulta diaria del médico forense. Se trata de procesos que en principio no revisten gravedad, pero que resultan muy invalidantes, pues dan lugar a una disminución de la actividad del lesionado en todos los ámbitos de la vida diaria. Basándonos en esto, presentamos un estudio con una muestra de 50 lesionados, aquejados de cervicalgias agudas de origen traumático, y determinamos los principales rasgos de personalidad de cada uno de ellos utilizando el Cuestionario EPQ-A de Eysenck para la valoración de la personalidad y el Inventario Multidimencional de Dolor West-Haven-Yale (WHYMPI). Los resultados obtenidos indican que las mujeres con dolor cervical postraumático presentan mayores grados de neuroticismo y menores de sinceridad, mientras que los hombres presentan igualmente una baja sinceridad. En cuanto a la valoración del dolor, los resultados muestran que conforme reciben tratamiento experimentan una disminución en la gravedad del dolor y en la influencia del dolor en la vida diaria.
\end{abstract}

Palabras clave: Dolor cervical postraumático. Personalidad. Valoración medico-legal.

\begin{abstract}
Posttraumatic cervical pain, is one of the most common pathologies in the daily consultation of forensic doctor. These processes are in principle not serious, but resulting in disability, which leads to a decreased activity of the injured person in all the areas of daily life. Based on this, we present a study with a sample of 50 injured persons, suffering from acute cervical pain of traumatic origin and we determine the principal feature of personality of each one of them, using the Questionnaire EPQ-A of Eysenck for the valuation of the personality and a Multidimensional Pain Inventory West-Haven-Yale (WHYMPI). From the obtained results we determine that women with posttraumatic cervical pain present mayor levels of neuroticism and minors of sincerity, whereas men present similar low levels of sincerity. As for the valuation of the pain, the results indicate us that treated accordingly, experience a decrease in both the severity of pain as a decrease in the influence of pain on daily life of the injured person.
\end{abstract}

Key words: Cervical posttraumatic pain. Personality. Valuation legal doctor.

\section{Introducción}

Resulta muy frecuente, en la práctica habitual del médico forense, la realización de valoraciones del daño corporal relacionadas con dolor cervical. Se trata de un cuadro complejo, generalmente no grave desde el punto de vista clínico, pero con una alta prevalencia, y además sin pruebas diagnósticas o complementarias objetivas que faciliten la labor pericial.
Aunque hay autores que indican la ausencia de una definición exacta y precisa de la cervicalgia ${ }^{1}$, sí podemos concretar que se trata de un dolor localizado en el cuello, que suele acompañarse de impotencia funcional para los movimientos de flexo-extensión, rotación y lateralización, y que puede irradiar a los hombros y la espalda. Otros autores establecen el concepto de una manera mucho más genérica, indicando que es un dolor localizado entre el occipucio y la tercera vértebra dorsal.

\section{Casado Blanco ${ }^{1}$ \\ M. Ibáñez Bernáldez ${ }^{2}$ \\ A. Raya Isla ${ }^{3}$ \\ P. Mata Ron ${ }^{4}$}

${ }^{1}$ Doctor en Medicina.

Especialista en Medicina Legal y Forense. Médico

Forense. Instituto de Medicina Legal de Badajoz.

Facultad de Medicina

UEX. Badajoz.

${ }^{2}$ Doctora en Medicina.

Médico Forense. Instituto de Medicina Legal de Badajoz. Facultad de Medicina UEX. Badajoz.

${ }^{3}$ Médico Forense.

Instituto de Medicina Legal de Badajoz.

Facultad de Medicina UEX. Badajoz.

${ }^{4}$ Especialista en Medicina Legal y Forense. Médico

Forense. Instituto de Medicina Legal de Badajoz. Facultad de Medicina UEX. Badajoz.

\section{Correspondencia:}

Mariano Casado Blanco. Instituto de Medicina Legal de Badajoz. Campus Universitario. Avda. de Elvas s/n.

06071 Badajoz.

E-mail: mariano.casado@ justicia.es

Fecha de recepción: 15. JUL. 2013

Fecha de aceptación: 04. DIC. 2013 
La causa más frecuente con que nos encontramos en el campo médico-legal es de tipo traumático, secundaria a movimientos bruscos del cuello, que conllevan pequeños desgarros músculo-ligamentarios. Es lo que se conoce como esguince, "latigazo" cervical o whiplash, siendo este último el término más referenciado en todas las publicaciones internacionales, y "palabra clave" en las búsquedas informatizadas. El mecanismo más frecuente es la flexo-extensión brusca del cuello por aceleración o desaceleración, como ocurre en los accidentes de tráfico, en general como consecuencia de mecanismos de alcance.

El término whiplash fue mencionado por primera vez por Crowe en 1928, en un estudio sobre ocho casos de traumatismo cervical en accidentes de circulación. No obstante, la generalización del uso de este término es objeto de crítica, pues se introduce un mecanismo de lesión como concepto clínico. Para evitar esta polémica se acuñó la denominación whiplash associated disorders (WAD), que aglutina las consecuencias derivadas del mecanismo de lesión de tipo whiplash: dolor cervical, cefalea, mareos, contractura muscular, rigidez, etc. WAD viene a representar el concepto clínico de síndrome postraumático cervical, recogido en el baremo español por el RDL 8/2004, de uso obligado en la valoración actual de las lesiones debidas a accidente de vehículo a motor.

Existen otras causas de cervicalgia, aunque no traumática, como son las patologías artrósicas-degenerativas, las posturas inadecuadas, las ocasionadas por sobrecarga muscular en determinadas profesiones, y las debidas a procesos de estrés o a cuadros de carácter psicoorgánico².

En otro orden de cuestiones ${ }^{3}$, la enfermedad no es una "cosa", no es algo por sí misma; la enfermedad es un proceso dinámico causado por la interacción de varios factores. De acuerdo con esta idea, el cuerpo y la mente no son formas separadas, sino que juntas constituyen el ser humano. De ahí la importancia de los factores biológicos en las emociones y en el comportamiento, de la misma manera en que los factores psicológicos y sociales afectan al curso de la enfermedad, lo que sin duda confirma el concepto de unitarismo del ser humano.

Centrándonos en los factores psicológicos, es evidente que hay una relación directa y que estos factores influyen en el estado físico del paciente; es lo que clásicamente se denominaba "trastornos psicosomáticos", término que la psiquiatría se encargó de desterrar de las clasificaciones internacionales, siendo sustituido por los "factores psicológicos que afectan al estado físico" del DSM-IV4 o los "factores psicológicos y del comportamiento en trastornos o enfermedades clasificados en otro lugar" de la categoría F54 de la CIE-105, que en el DSM5 se siguen manteniendo ${ }^{6}$.

Concretando un poco más, Millon y Roger ${ }^{7}$ establecían la relación entre personalidad y desarrollo, mantenimiento y tratamiento de los trastornos clínicos especificados en el eje I del DSM de los trastornos mentales, y trataban también la posibilidad de establecer una relación entre la personalidad y las enfermedades médicas.

Sin embargo, si de personalidad hemos de hablar, podemos plantearnos la influencia que pudiera tener una bioquímica deficiente. De este modo, en los trabajos de Cloninger y Svrakic $^{8}$ se proponen relaciones directas entre sustancias químicas y características de la personalidad (p. ej., la serotonina relacionada con la evitación del dolor, la dopamina con la búsqueda de novedad, y la noradrenalina con la dependencia de la recompensa).

Directamente conectado con el proceso patológico aparece el dolor, el cual a su vez se ha relacionado con diversos aspectos psicológicos como son los rasgos ciclotímicos, histéricos (exageración de síntomas) u obsesivos (cadenas de pensamientos anticipatorios), el alcoholismo, la depresión, el abuso sexual, los antecedentes de abuso infantil o de experiencias infantiles negativas frecuentes, y un menor nivel educativo; muchos de ellos correlacionados con menores umbrales de dolor o con la utilización de estrategias inadecuadas de afrontamiento del dolor ${ }^{9,10}$.

Por último, en esta exposición, nos podemos plantear si existe concordancia entre los rasgos de personalidad del paciente y la influencia de éstos en el dolor. A este respecto hay estudios variados. Así, algunas investigaciones determinan que las psicopatologías más relacionadas con el dolor crónico son la depresión y la ansiedad ${ }^{11}$, así como los perfiles histéricos, neuróticos e hipocondríacos ${ }^{12}$. También se ha descrito que la depresión, la ansiedad y la ira (esta última en menor medida) tienen una relación directamente proporcional entre el origen, el mantenimiento y la exacerbación del dolor ${ }^{13}$. Sin embargo, otros autores plantean serias incertidumbres acerca de que el dolor pueda ser atribuido a un tipo de personalidad premórbida $^{14}$.

Otras líneas de investigación, como bien recogen Infante Vargas et al. ${ }^{15}$, más bien apuntan a que la personalidad no es un precursor del dolor, sino que determina o media en parte el grado de incapacidad, cronificación y sufrimiento ${ }^{16}$. 


\section{Material y método}

Partiendo de lo anteriormente expuesto y de la alta incidencia de dolores cervicales en la consulta médica forense, generalmente a consecuencia de accidentes de tráfico, y teniendo en cuenta, como ya se ha indicado, que se trata de procesos clínicos que en principio no revisten gravedad pero que resultan muy invalidantes y provocan alteraciones en la vida diaria de los lesionados, y por otro lado tratando de determinar si los lesionados con estos cuadros clínicos podrían presentar unas características psicopatológicas, nos planteamos diseñar un estudio en el que se pudieran determinar rasgos de personalidad en los lesionados con cervicalgia y analizar las características del cuadro doloroso y su influencia en las actividades de la vida diaria, tanto al inicio como en el momento de emitir el correspondiente informe de sanidad. Basándonos en esto y siguiendo el mismo esquema utilizado por otros autores ${ }^{17}$ con pacientes con cervicalgias crónicas no traumáticas, realizamos el estudio con 50 lesionados, aquejados de cervicalgias agudas tras haber sufrido algún tipo de evento traumático, y determinamos los principales rasgos de personalidad de cada uno de ellos utilizando el Cuestionario EPQ-A de Eysenck para la valoración de la personalidad y el Inventario Multidimencional de Dolor West-Haven-Yale (WHYMPI).

EI EPQ-A ${ }^{18}$ es una escala que consta de 94 ítems que hacen referencia a distintas maneras de pensar y sentir, con dos posibles respuestas (sí/no), y a partir de ellos pueden obtenerse cuatro valores referidos a neuroticismo $(N)$, extroversión $(E)$, psicoticismo $(P)$ y sinceridad (S). A cada uno de estos factores de personalidad, según Eysenck, se asocia una serie de rasgos psíquicos de la persona.

Por su parte, el WHYMPI ${ }^{19}$ consta de 52 ítems con siete respuestas cada uno, valoradas de 0 a 6 y divididas en tres partes; la primera hace referencia al dolor del individuo y a cómo afecta a su vida, la segunda estudia el apoyo y la respuesta familiar ante el dolor, y la tercera se refiere a las actividades cotidianas que el individuo realiza.

De los 50 lesionados valorados, 36 eran mujeres (72\%) y 14 hombres (28\%), con una edad media de 35 años las mujeres y de 39 años los hombres, con un intervalo de edad de 19 a 75 años.

Respecto a las actividades laborales/profesionales, el $36 \%$ eran amas de casa, un $6 \%$ eran estudiantes, un $6 \%$ personas sin estudios, un $24 \%$ con estudios medios, un $6 \%$ con estudios superiores, un $4 \%$ jubilados y un $18 \%$ personas que manifestaban estar en paro o desempleados.
En cuanto al origen del dolor cervical, el $92 \%$ de los lesionados lo eran como consecuencia de accidentes de tráfico y el $8 \%$ por otros motivos igualmente traumáticos (accidentes laborales o agresiones).

Todos ellos estaban bajo control del médico traumatólogo de la correspondiente compañía de seguros, quien derivaba al lesionado al médico rehabilitador y éste prescribía la correspondiente fisioterapia, que en líneas generales y según se establece por protocolo consistía en masoterapia, tracciones cervicales y aplicación de microondas.

La valoración del EPQ-A se realizó durante la primera visita realizada a la consulta médica forense, mientras que el inventario WHYMI se cumplimentó igualmente durante la primera visita y en el momento de emitir el informe de sanidad, por haber alcanzado la curación o la estabilización de la lesión.

Los datos fueron sometidos al correspondiente análisis estadístico utilizando el programa SPSS para Windows, versión 10.0. Para estudiar la asociación entre una variable continua y una dicotómica se aplicó la prueba t de Student (para variables cualitativas que seguían una distribución normal) y se consideró un nivel de significación de $p<0,025$.

\section{Resultados}

Al valorar a los 50 lesionados mediante el EPQ-A se obtuvieron las cuatro puntuaciones correspondientes a cada uno de los valores analizados: neuroticismo o emocionabilidad, con una media de 16,8 (DE: 5,3); extroversión, con una media de 12,3 (DE: 4,3); psicoticismo o dureza, con una media de 3,0 (DE: 2,6); y sinceridad, con una media de 8,1 (DE: 4,5). Estos resultados fueron comparados con los que obtuvo Eysenck en su trabajo con población general, tanto hombres como mujeres, mediante la t de Student para una determinada prueba. Los resultados se exponen en la Tabla 1. De ella, podemos determinar como dato más significativo que las mujeres con cervicalgias agudas presentan mayores grados de neuroticismo y menores de sinceridad que la población general. Del mismo modo, los hombres con cervicalgia aguda son igualmente menos sinceros (Tabla 2).

Por otra parte, mediante el WHYMPI se analizaron sucesivamente las características del dolor y su influencia en las actividades de la vida del lesionado, la respuesta ante ese dolor de las personas cercanas al lesionado, y su interferencia en las actividades de la vida diaria. Como ya se ha indicado, este cuestionario se valoró en dos ocasiones, durante la primera 
Tabla 1.

Comparación de nuestros resultados en el EPQ-A con

los obtenidos por Eysenck

(1997) sobre población general, en mujeres.

\begin{tabular}{ccccc}
\hline Mujeres & $\begin{array}{c}\text { Grupo en estudio } \\
\text { Media (DE) }\end{array}$ & $\begin{array}{c}\text { Valores de referencia } \\
\text { Media (DE) }\end{array}$ & $\mathbf{t}$ & $\mathbf{p}$ \\
\hline EPQ-N & $16,8(5,3)$ & $14,5(5,2)$ & 2,459 & 0,026 \\
EPQ-E & $12,3(4,3)$ & $11,4(4,4)$ & 0,558 & NS \\
EPQ-P & $3,0(2,6)$ & $2,2(2,4)$ & 1,904 & NS \\
EPQ-S & $8,1(4,5)$ & $11,9(4,3)$ & $-9,073$ & 0,000 \\
\hline
\end{tabular}

Tabla 2.

Comparación de nuestros resultados en el EPQ-A con

los obtenidos por Eysenck

(1997) sobre población general, en hombres.

\begin{tabular}{lcccc}
\hline Hombres & $\begin{array}{c}\text { Grupo en estudio } \\
\text { Media (DE) }\end{array}$ & $\begin{array}{c}\text { Valores de referencia } \\
\text { Media (DE) }\end{array}$ & $\mathbf{t}$ & $\mathbf{p}$ \\
\hline EPQ-N & $12,6(5,3)$ & $11,6(5,3)$ & 1,035 & NS \\
EPQ-E & $12,1(4,4)$ & $12,0(4,0)$ & 0,025 & NS \\
EPQ-P & $2,6(2,3)$ & $2,7(3,1)$ & $-0,439$ & NS \\
EPQ-S & $7,8(4,3)$ & $10,3(4,9)$ & $-3,348$ & 0,002 \\
\hline
\end{tabular}

visita a la consulta y al final del proceso previamente a emitir el correspondiente informe de sanidad, fuera por curación o por estabilización de la lesión. Desde el punto de vista estadístico, igualmente se utilizó la prueba t de Student.

Como puede apreciarse en la Tabla 3, entre la primera valoración y la última se observa una disminución en la intensidad del dolor, en la interferencia de éste en la vida diaria del lesionado y en su estado de ánimo, con una marcada mejoría del autocontrol y del impacto general del dolor en la vida del lesionado.

De igual modo, en la segunda valoración se aprecia una disminución en la sensación de rechazo por personas próximas y se sienten más atendidos en sus problemas. También resulta evidente que aumentan las actividades de ocio, las actividades sociales y, en definitiva, todo tipo de actividades de la vida diaria.

\section{Discusión}

El dolor cervical es un problema relativamente frecuente en nuestra sociedad. El síndrome del latigazo cervical se produce por un traumatismo cervical causado por una extensión violenta del cuello. De acuerdo con la Quebec Task Force on Whiplash-As- sociated Disorders (WAD) ${ }^{20}$, el síndrome de latigazo cervical se define como un conjunto de síntomas que aparecen después de un mecanismo de aceleracióndesaceleración con transferencia de energía al cuello, secundario a colisiones entre vehículos, pero que también puede ocurrir tras zambullidas en el agua o en otras circunstancias desafortunadas. De entre todos, los casos relacionados con dolores cervicales como consecuencia de accidentes de tráfico son muy frecuentes. De hecho, en la consulta médica forense constituye la causa más habitual de valoración del daño corporal.

Siguiendo el modelo biopsicosocial, el curso natural del cuadro de cervicalgia postraumática está determinado por un origen multifactorial, en cuyo desarrollo están implicados diferentes factores de riesgo, ya sean físicos, psicosociales e incluso individuales ${ }^{21}$, razón por la que algunos estudios sobre este tema se basan en dicho modelo ${ }^{22}$.

En líneas generales partimos de un dato, y es que la prevalencia del dolor cervical es más alta entre la población femenina, como así lo determinan la mayoría de los estudios revisados ${ }^{23,24}$, coincidentes con el nuestro.

Con respecto a la cervicalgia postraumática, en nuestro estudio, a pesar de la limitada muestra de 


\begin{tabular}{lcccc}
\hline & Inicio del tratamiento & Final del tratamiento & t & $\boldsymbol{p}$ \\
\hline Interferencia & $2,4(1,4)$ & $2,1(1,4)$ & 5,924 & 0,000 \\
Apoyo & $3,3(1,8)$ & $3,3(1,8)$ & 0,231 & $\mathrm{NS}$ \\
Severidad & $3,2(1,4)$ & $1,7(1,3)$ & 14,482 & 0,000 \\
Autocontrol & $4,0(1,3)$ & $4,4(1,3)$ & $-3,584$ & 0,000 \\
Estado de ánimo & $2,7(1,3)$ & $2,0(1,1)$ & 7,309 & 0,000 \\
Total 1 & $3,1(0,8)$ & $2,7(0,7)$ & 8,955 & 0,000 \\
Rechazo & $1,6(1,0)$ & $1,3(1,1)$ & 5,172 & 0,000 \\
Atención & $3,1(1,7)$ & $3,2(1,6)$ & $-1,006$ & $\mathrm{NS}$ \\
Distracción & $2,1(1,2)$ & $2,0(1,1)$ & 1,839 & $\mathrm{NS}$ \\
Total 2 & $2,3(1,0)$ & $2,1(0,9)$ & 2,830 & 0,005 \\
Activ. domiciliarias & $2,5(1,7)$ & $2,5(1,6)$ & $-0,306$ & $\mathrm{NS}$ \\
Activ. aire libre & $2,1(1,0)$ & $2,2(1,0)$ & $-3,168$ & 0,002 \\
Activ. fuera casa & $2,8(1,2)$ & $3,0(1,2)$ & $-2,553$ & 0,012 \\
Activ. sociales & $4,0(1,8)$ & $4,2(1,7)$ & $-3,487$ & 0,001 \\
Total 3 & $2,8(1,0)$ & $3,0(1,0)$ & $-4,445$ & 0,000 \\
\hline
\end{tabular}

Tabla 3.

Resultados globales del WHYMPI. pacientes, igualmente se evidencia una alta prevalencia $(72 \%)$ de mujeres que presentan sintomatología postraumática, dato que coincide con algunos otros trabajos que pueden encontrarse en la literatura médica ${ }^{25,26}$. Ante este dato podrían plantearse varias cuestiones. En primer lugar, aun teniendo en cuenta que en la mayoría de los trabajos revisados la prevalencia de mujeres es más alta, no hay evidencia suficiente de que el sexo sea un factor relacionado con la evolución clínica del síndrome de latigazo cervical o de cervicalgia postraumática ${ }^{26}$. Igualmente, otros dos datos podrían ser interesantes para fijar la relación entre traumatismo, cervicalgia y personalidad. Por una parte, determinar la existencia de patologías previas cervicales que pudieran condicionar la sintomatología posterior, sobre lo cual hay trabajos, como el de Wynne-Jones ${ }^{27}$, en que no parece que este dato sea un factor a tener en cuenta en la clínica postraumática. Y por otro lado, tampoco hay evidencias claras de que el consumo previo de psicofármacos se relacione con una peor evolución ${ }^{28,29}$, lo que lleva a pensar que presentar una enfermedad psicológica antes del accidente no es un factor que influya en la evolución clínica.

De los resultados también podemos destacar que las mujeres con cervicalgia postraumática, además de tener un mayor nivel de neuroticismo que los hombres con similar origen de dolor cervical, tienen también un mayor nivel de neuroticismo que la población general. Esto provoca en las mujeres una serie de rasgos asociados, tales como mayor ansiedad, mayores sentimientos de culpa, baja autoestima, aumento de tensión, inestabilidad emocional, más cambios de humor, tendencia a la depresión, problemas para conciliar el sueño y disposición a presentar quejas de tipo psicosomático.

Teniendo en cuenta lo anterior y valorando los factores individuales, que es el objeto de este estudio y referidos concretamente a la personalidad, nuestros resultados determinan que en la valoración de los accidentados estudiados sí aparecen alteraciones según el EPQ-A, sobre todo en las mujeres, que presentan mayores niveles de neuroticismo e incluso algo mayores que en la población con cervicalgia de tipo crónico no traumática ${ }^{17}$, y tanto los hombres como las mujeres presentan una baja sinceridad.

Además, parece evidente que la forma de percibir el dolor puede estar influida por la personalidad del accidentado. Es difícil hacer una valoración real del dolor, pues nos basamos en apreciaciones subjetivas del propio lesionado y en sus experiencias previas de dolor. Es decir, sólo podemos juzgar el dolor por el conocimiento del lesionado y sus antecedentes, y ello 
varía dependiendo de la personalidad del paciente. Lo cierto es que algunas características de la personalidad dan lugar a una actitud desfavorable hacia el dolor. Así, no resulta difícil entender que una personalidad de este tipo se enfrente al dolor con una gran ansiedad y con una fuerte predisposición a exagerar el impacto del hecho traumático, contribuyendo ello a acentuar la vivencia subjetiva del dolor como una experiencia displacentera. De esta forma podríamos indicar que los rasgos de personalidad parecen poder distorsionar de manera notable la percepción del dolor. Esto puede guardar relación con el hecho de que quienes han sufrido un traumatismo cervical o síndrome de latigazo cervical pudieran desarrollar síntomas psicológicos después del accidente, posiblemente en respuesta al estrés postraumático, que sí podría considerarse que influye en la evolución clínica, tal como indican diferentes trabajos ${ }^{30,31}$.

Otros autores ${ }^{32}$ han defendido el papel de los factores de personalidad como causa contribuyente al dolor muscular, al condicionar una variación en la experiencia de dolor básicamente a través de la inducción de sentimientos de ansiedad y disforia. De acuerdo con Cloninger $^{33}$, los extremos de temperamento pueden predisponer a los sujetos a la conducta inadaptada, básicamente relacionada con la aparición de trastornos emocionales como la depresión y la ansiedad.

Como última cuestión, y según lo planteado en la introducción del trabajo, podemos discutir acerca de la relación o la influencia que podría haber entre alteraciones psicológicas (neuroticismo) y alteracio- nes biológicas en referencia al cuadro de cervicalgia aguda. De forma muy básica, podemos señalar que existe esta relación. El propio Eysenck estudió las dimensiones de la personalidad desde el punto de vista de sus bases fisiológicas, y asoció sus factores de psicoticismo, neuroticismo y extroversión con variaciones de las concentraciones de dopamina, serotonina y noradrenalina, respectivamente ${ }^{34}$, cuestiones que pueden plantear discusión acerca de la existencia de bases biológicas respecto a las diferencias individuales genéticas, presencia de polimorfismos genéticos o incluso alteraciones en el metabolismo de la serotonina.

\section{Conclusiones}

Puesto que los pacientes con cervicalgias postraumáticas presentan un mayor grado de neuroticismo, sobre todo las mujeres, planteamos que este nivel podría actuar distorsionando la percepción del dolor, actuando de forma desproporcionada al cuadro clínico y con manifestaciones básicamente psicopatológicas, provocando ansiedad y depresión, además de una exageración del dolor. También consideramos que la personalidad influye en la evolución del cuadro clínico de cervicalgia aguda, si no de una forma objetiva sí con la presencia de factores subjetivos.

Los autores declaran no tener conflicto de intereses.

\section{Bibliografía}

1. Meseguer Henarejos AB, Medina i Mirapeix F, Cánovas Gascón JJ, Esteban Argente I, Torres Vaquero Al, Alcántara F. Fisioterapia. 2000;22(2):4-12. (Ejemplar dedicado a Cervicalgia.)

2. Vázquez A. Cervico-braquialgia. En: Oliván AS, Pinillos MA, Agorreta J, Rubio T, editores. Guía de Urgencias Hospital de Navarra 1999. Hoechst Marion Roussel. 1999;267-8.

3. Molina JA. Underting the biopsychosocial model. World Psychiatric Association and APA Regional Meeting New York City; New York, 1981.

4. American Psychiatric Association. Diagnostic and Statistical Manual of Mental Disorders, 4th ed. Washington DC: American Psychiatric Association; 1994.

5. Miralles Alonso L, Otin Llop R, Rojo Rodes JE. Factores psicológicos que afectan al estado físico. Medicine. 2003;8(105):5654-64.
6. American Psychiatric Association. Diagnostic and Statistical Manual of Mental Disorders. 5th ed. DSM5. Washington, DC; 2013.

7. Millon T, Roger D. Trastornos de personalidad: más allá del DSM-IV. Barcelona: Masson; 1998.

8. Cloninger CR, Svrakic DM, Pryzbeck TR. A psychobiological model of temperament and character. Archives of General Psychiatry. 1993;50:975-90.

9. Moix J. Análisis de los factores psicológicos moduladores del dolor crónico benigno. Anuario de Psicología. 2005;36(1):37-60.

10. Valls $C$. Diagnóstico diferencial del dolor y de la fibromialgia. Anuario de Psicología. 2008;39(1):87-92.

11. Mayr M, Hogler S, Ghedina W, Berck K. Low back and psychiatric disorders. Lancet. 2003;361:9356. 
12. Breslau N, Chilcoat HD, Andreski P. Further evidence on the link between migraine and neuroticism. Neurology. 1996;47:563-667.

13. Truyols M, Pérez J, Medinas M, Palmer A, Sesé A. Aspectos psicológicos relevantes en el estudio y el tratamiento del dolor crónico. Clínica y Salud. 2008;19(3):295-320.

14. Serrano M, Cañas A, Serrano C, García PI, Caballero J. Evaluación psíquica del dolor. Dolor. 2000;17:57-70.

15. Infante Vargas P, García Parés G, Moix Queraltó J, Cañellas Arsego M, Subirá Álvarez S. Personalidad y dolor. Psiquis. 2005;26(3):93-100.

16. Dersh J, Polatin PB, Gatchel RJ. Chronic pain and psychopathology: research findings and theoretical considerations. Psychosom Med. 2002;64(5):77386.

17. Montes Doncel E. Personalidad y psicopatología como factores moduladores de la percepción del dolor en pacientes con cervicalgia. Tesis doctoral. Badajoz: Universidad de Extremadura; 2002.

18. Eysenck HJ, Eysenck SBG. Cuestionario de personalidad para adultos (EPQ-A). Publicaciones de psicología aplicada, 8ª edición. Madrid: TEA ediciones, 1997.

19. Kerns RD, Turk DC, Rudy TE. The West Haven-Yale Multidimensional Pain Inventory (WHYMPI). Pain. 1985;23:345-56.

20. Spitzer WO, Skovron ML, Salmi LR, Cassidy JD, Duranceau J, Suissa S, et al. Scientific monograph of the Quebec Task Force on Whiplash-Associated Disorders: redefining "whiplash" and its management. Spine. 1995;20(8 Suppl):1-73.

21. Ariëns GAM, Borghounts JAJ, Koes BW. Neck pain En: Cronbie IK, editor. The epidemiology of pain. Seattle: IASP Press; 1999.

22. Scholten-Peeters GG, Verhagen AP, Bekkering GE, van der Windt DA, Barnsley L, Oostendorp RA, et al. Prognostic factors of whiplash-associated disorders: a systematic review of prospective cohort studies. Pain. 2003;104(1-2):303-22.

23. Lau EMC, Sham A, Wong KC. The prevalence of and risk factors for neck pain in Hong Kong Chinese. $J$ Public Health Med. 1996;18(4):396-9.
24. Croft PR, Lewis M, Papageorgiou AC, Thomas E, Jayson MIV, Macfarlane GJ, et al. Risk factors for neck pain: a longitudinal study in the general population. Pain. 2001;93:317-25.

25. Hendriks EJ, Scholten-Peeters GG, Van der Windt DA, Neeleman-van der Steen CW, Oostendorp RA, Verhagen AP. Prognostic factors for poor recovery in acute whiplash patients. Pain. 2005;114(3):40816.

26. Pleguezuelos E, Pérez ME, Guirao L, Palomera E, Moreno E, Samitier B. Factores relacionados con la evolución del síndrome del latigazo cervical. Med Clin. 2008;131(6):211-5.

27. Wynne-Jones G, Jones GT, Atherton K, Wiles NJ, Silman AJ, Macfarlane GJ. Comment on Hendriks, et al.: Prognostic factors for poor recovery in acute whiplash patients. Pain. 2005;119(1-3):247-8.

28. Radanov B, Bicik I, Dvorak J, Antinnes J, von Schulthess GK, Buck A. Relation between neuropsychological and neuroimaging findings in patients with late whiplash syndrome. J Neurol Neurosurg Psychiatry. 1999;66(4):485-9.

29. Mayou R, Radanov BP. Whiplash neck injury. Journal of Psychosomatic Research. 1996;40(5):461-74.

30. Sterling M, Kenardy J. The relationship between sensory and sympathetic nervous system changes and posttraumatic stress reaction following whiplash injury - a prospective study. Journal of Psychosomatic Research. 2006;60(4):387-93.

31. Cassidy JD, Carroll LJ, Pierre Côté P, Lemstra $M$, Berglund A, Nygren A. Effect of eliminating compensation for pain and suffering on the outcome of insurance claims for whiplash injury. N Eng/ J Med. 2000;342:1179-86.

32. Svebak S, Mykletun RJ, Bru E. Connections between musculoskeletal complaints and personality. Tidsskr Nor Laegeforen. 1994;114:685-8.

33. Cloninger CR. A systematic method for clinical description and classification of personality variants. Archives of General Psychiatty. 1997;44:573-88.

34. Lester DA. Neurotransmitter basis for Eysenck's theory of personality. Psicol Rep. 1989;64(1):18990 . 